\title{
Frequent $T$ cell responses against immunogenic targets in lung cancer patients for targeted immunotherapy
}

\author{
ANNA BABIAK $^{1 *}$, MAX STEINHAUSER $^{1 *}$, MARLIES GÖTZ $^{1}$, CORNELIA HERBST $^{1}$, \\ HARTMUT DÖHNER $^{1}$ and JOCHEN GREINER ${ }^{1,2}$ \\ ${ }^{1}$ Department of Internal Medicine III, University of Ulm, D-89081 Ulm; \\ ${ }^{2}$ Department of Internal Medicine, Diakonie Hospital Stuttgart, D-70176 Stuttgart, Germany
}

Received July 8, 2013; Accepted August 26, 2013

DOI: $10.3892 /$ or.2013.2804

\begin{abstract}
To date, lung cancer is one of the leading causes of cancer mortality with short overall survival despite adequate therapy. New immunotherapeutic strategies using peptides derived from tumor-associated antigens (TAAs) can induce a specific cytotoxic $\mathrm{T}$ cell (CTL) response leading to a targeted tumor cell death. In the present study, we addressed whether there are further significant immunogenic candidate targets that may induce strong immune reactions with a high frequency in lung cancer patients eligible for cellular immunotherapeutic approaches, such as in a polyvalent vaccination approach. In this study, we investigated specific CTL responses of 14 HLA-A*0201-positive patients (of 33 screened patients) with non-small cell lung cancer (NSCLC; $n=12$ ) or small cell lung cancer (SCLC; $n=2$ ) against several known and novel TAA-derived peptides from lung cancer and/or other tumor entities, by measuring granzyme B (GrB) and/ or interferon $\gamma($ IFN $\gamma)$ secretion using enzyme-linked immunospot (ELISpot) analysis. Specific T cell responses could be detected for hTERT (4/13), two MAGE-A3-derived peptides (4/13 and 3/13, respectively), RHAMM (4/14), PRAME (8/14), G250 (7/12), survivin (3/13), HER2 (5/10) and WT1 (2/14), but also novel epitopes derived from Aurora kinase A (4/13) and B (5/13). Additionally, simultaneous CTL responses against the different peptides were examined and specific $\mathrm{T}$ cell responses against at least one of these TAAs could be detected in 13/14 (93\%) patients. It could be shown that all patients with immune reactions against RHAMM and hTERT showed also immune responses against PRAME. Furthermore, patients with CTL responses against the Aurora kinase A peptide (Aura A1) also
\end{abstract}

Correspondence to: Professor Jochen Greiner, Department of Internal Medicine III, University of Ulm, Albert-Einstein-Allee 23, D-89081 Ulm, Germany

E-mail: jochen.greiner@uniklinik-ulm.de

Department of Hematology and Oncology, Diakonie-Klinikum Stuttgart, Rosenbergstrasse 38, D-70176 Stuttgart, Germany

E-mail: greiner@diak-stuttgart.de

${ }^{*}$ Contributed equally

Key words: immunotherapy, lung cancer, tumor-associated antigens demonstrated a response against the Aurora kinase B peptide (Aura B1). Taken together, we showed that these TAA-derived peptides induce frequent specific $\mathrm{T}$ cell responses in patients with metastatic lung cancer and are, therefore, novel candidates for targeted immunotherapies and polyvalent approaches.

\section{Introduction}

In recent years, targeted therapies in the treatment of lung cancer have been implemented. Aside from thyrosine kinase inhibitors targeting EGFR and EML4-ALK, such as gefitinib, erlotinib and crizotinib as well as monoclonal antibodies against VEGFR (bevacizumab), specific therapies using cellular immunity have also been established or are in clinical examination (1-3). It is known that $\mathrm{T}$ cell mediated immune responses can play a crucial role in tumor defense; tumor cells can be efficiently killed by specific $\mathrm{T}$ cells of the immune system (4). T cell activating antibodies against CTLA-4 (ipilimumab) and against the inhibitory $\mathrm{T}$ cell receptor programmed death-1 (PD-1) showed promising results against lung cancer in clinical testing $(5,6)$. Active immunotherapies induce a specific immune response by recognizing epitopes being expressed in the tumor cell surface. Due to their association with tumor cells, these epitopes are called tumor-associated antigens (TAAs). TAAs are exclusively expressed and/or overexpressed in tumor cells, and are therefore useful to induce a tumor-specific immune response without harming healthy organs lacking TAA-expression (7-9). In the past years, several TAAs in hematological and oncological neoplasms have been identified. These TAAs are proteins or receptors playing an important role in cell cycle, proliferation and differentiation $(10,11)$.

The primary objective of active cancer immunotherapy is the induction of targeted cell death by activated specific CD8 ${ }^{+}$ cytotoxic T cells (CTLs) $(12,13)$. These activated CTLs recognize tumor cells that display the corresponding peptide derived from TAAs by MHC class I molecule on their surface which leads to a differentiation and expansion of CTLs and finally results in induction of lyses of the peptide presenting cells by the release of cytotoxins including perforin, granzymes and granulysin (14-16). In these processes, interferon $\gamma$ (IFN $\gamma$ ) plays a crucial role in promoting various immunoregulatory effects.

Previously, peptide vaccines against different TAAs such as MAGE-A3 and hTERT have been tested in lung cancer 
patients and phase III trials are ongoing (17-19). Tumor vaccination seems to be a promising strategy especially in situations of reduced lung tumor load, i.e. in maintenance therapy after induction chemotherapy or for patients with a low tumor load in further treatment lines $(2,20)$. Aside from single peptide vaccination, peptide mixtures may be a potent strategy inducing cancer-defending immunity. It was demonstrated that immune responses to multipeptide vaccine IMA901 were associated with prolonged overall survival in patients with advanced renal cell cancer (21). Therefore, it is important to identify TAA-derived peptides inducing CTL response in lung cancer that are potential candidates for developing potent single or polyvalent vaccines.

\section{Materials and methods}

Blood samples and preparation. A total of 33 patients were screened for HLA-A*0201 expression (see below) and subsequently analyses were performed in 14 HLA-A*0201-positive patients with diagnosed lung cancer, either non-small cell lung cancer (NSCLC; $n=12 ; 8$ adenocarcinoma and 3 squamous cell carcinoma, 1 sarcomatoid carcinoma) or small cell lung cancer (SCLC, $n=2$ ). Clinical characteristics of analyzed patients including age, tumor stage at time of diagnosis, histology, first line therapy and response to first-line therapy are shown in Table I. Additionally, mutation status concerning EGFR and EML4-ALK (if available) is shown. All patients underwent treatment at our Department of Internal Medicine. For blood analyses, an ethics proposal was filed and accepted by the Ethics Committee of the University of Ulm (application no. 09/10).

Pre-treatment peripheral blood [anti-coagulated with ethylenediaminetetraacetic acid (EDTA)] was obtained at date of diagnosis and in the course of following visitations during tumor therapy. For analyses described in these experiments we used blood samples from the date of diagnosis.

Ficoll density gradient centrifugation peripheral blood mononuclear cells (PBMCs) were resuspended in fetal calf

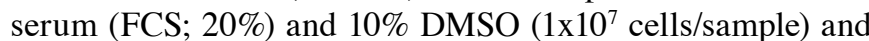
cryopreserved as previously described (22).

HLA-typing by flow cytometry. All peptides used in our experiments were HLA-A*0201 restricted. To identify HLA-A*0201 positive cells, flow cytometry was used (Fig. 1). Cells were incubated with an FITC labeled monoclonal anti-HLA-A*0201 antibody (BD, Heidelberg, Germany). As positive and negative control, the T2 and the K562 cell lines were used, respectively. After incubation at $4^{\circ} \mathrm{C}$ for $20 \mathrm{~min}$ in the dark and after washing twice, stained cells were analyzed by flow cytometry as previously described (23). From 33 screened patients, 14 were eligible for further experiments.

Peptides used in experiments. The peptides used in our experiments were derived from the well known TAAs RHAMM, PRAME, WT1, from hTERT, survivin, MAGE-A3, G250, HER2, Aurora kinase (Aura) A and B. Except for the peptides derived from Aurora kinases (Aura A1 and Aura B1) all peptides are well known $(11,24-26)$. The entire amino acid sequences of Aura A1 and Aura B1 were screened for HLA-A*0201 binding T cell epitopes using the algorithms of the SYFPEITHI (www. syfpeithi.de), the Rankpep (http://imed.med.ucm.es/tools) and
A

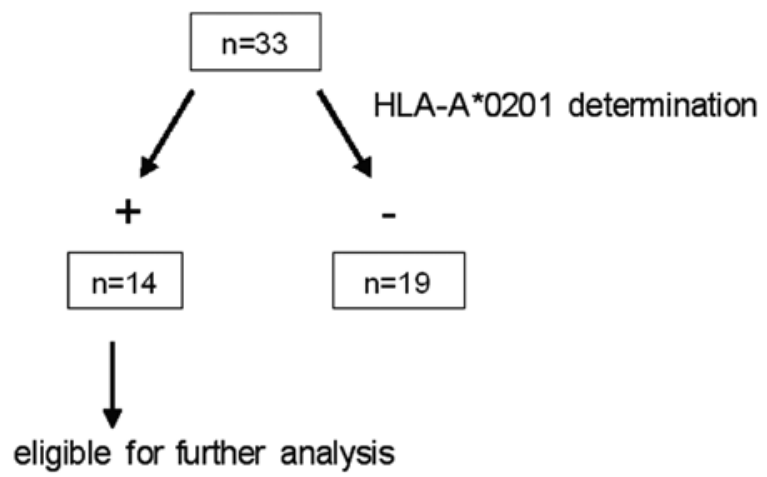

B
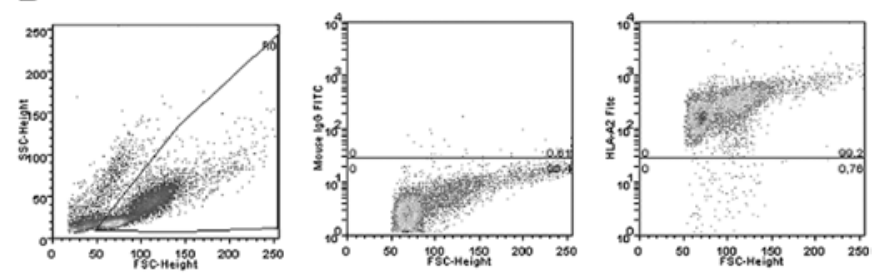

C
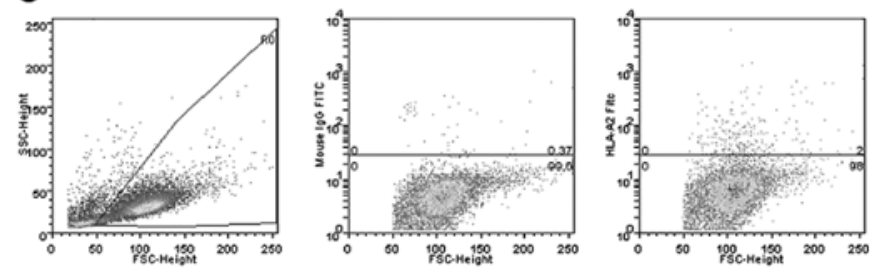

Figure 1. Screening of patients. (A) HLA-A*0201-positive patients for further experiments by FACS analysis. Thirty-three lung cancer patients were screened. Of these, 14 patients were positive and 19 were negative for HLA-A*0201. Exemplary FACS analysis of (B) an HLA-A*0201-positive and (C) an HLA-A*0201-negative patient.

the HLA-Bind (www-bimas.cit.nih.gov) software programs. For the novel antigens, $>10$ HLA-A*0201-binding peptides were predicted and these Aurora kinase-derived peptides were tested in 20 healthy volunteers and 14 lung cancer patients at primary diagnosis. The peptides were purchased from GL Biochem Ltd., Shanghai, China and Thermo Fisher Scientific, Ulm, Germany.

The sequences of the peptides used in our experiments are shown in Table II. Peptides from cytomegalovirus and influenza virus were used as positive controls due to their endemic occurrence (Table II).

Mixed lymphocyte peptide culture $(M L P C)$. PBMCs from patients and healthy volunteers were selected by magnetic beads through a magnetic-activated cell-sorting (MACS) column (Miltenyi Biotec, Bergisch Gladbach, Germany). CD8 antigen presenting cells (APCs) were irradiated with 30 Gy and pulsed for $2 \mathrm{~h}$ with either a TAA-derived peptide or a control peptide at a concentration of $20 \mu \mathrm{g} / \mathrm{ml}$. For all peptides, as well as for the control peptides, the same conditions and reagents were used as previously described (27).

ELISpot analysis. ELISpots were performed ex vivo on cultured patient cells and the T2 cell line as APCs. Briefly, the irradiated $\mathrm{CD}^{-}$fraction of autologous patient $\mathrm{PBMCs}$ 
Table I. Clinical characteristics of analyzed patients.

\begin{tabular}{cccccl}
\hline Patient & $\begin{array}{c}\text { Age } \\
\text { (years) }\end{array}$ & Diagnosis/stage ${ }^{\mathrm{a}}$ & Histology & $\begin{array}{c}\text { EGFR/ } \\
\text { EML4-ALK }\end{array}$ & \multicolumn{1}{c}{ Therapy $^{\mathrm{b}}$} \\
\hline 1 & 57 & NSCLC/IV & Squamous-cell & WT/neg. & Cisplatin/gemcitabine + radiaton \\
2 & 60 & NSCLC/IV & Adeno & WT/neg. & $\begin{array}{l}\text { Cisplatin/pemetrexed/bevacizumab } \\
\text { maintenance bevacizumab }\end{array}$ \\
3 & 67 & NSCLC/IIIA & Adeno & WT/neg. & Neoadjuvant radiochemotherapy \\
4 & 44 & NSCLC/IV & Adeno & WT/n.a. & No therapy \\
5 & 49 & NSCLC/IV & Adeno & WT/n.a. & Carboplatin/pemetrexed \\
6 & 64 & NSCLC/IB & Adeno & n.a. & Tumor resection \\
7 & 62 & SCLC/ED & & n.a. & Cisplatin/etoposide \\
8 & 78 & NSCLC/IV & Sarcomatoid & WT/n.a. & Not known \\
9 & 54 & NSCLC/IA & Squamous-cell & n.a. & Tumor resection \\
10 & 52 & NSCLC/IV & Squamous-cell & n.a. & Tumor resection \\
11 & 79 & NSCLC/IV & Adeno & WT/n.a. & Carboplatin/docetaxel \\
12 & 61 & NSCLC/IV & Adeno & WT/n.a. & Cisplatin/vinorelbine \\
13 & 80 & SCLC/ED & & n.a. & Topotecan \\
14 & 65 & NSCLC/IV & Adeno & Mut./neg. & Carboplatin/docetaxel/erlotinib \\
\hline
\end{tabular}

Fourteen patients with metastatic lung cancer were HLA-A2 positive and were analyzed by ELISpot analysis. NSCLC, non-small cell lung cancer; SCLC, small-cell lung cancer; ED, extensive disease; WT, wild-type; neg., negative; $n$.a., not analyzed; mut., mutated; PD, progressive

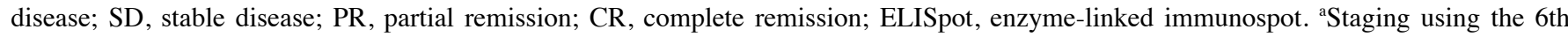

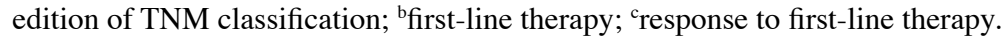

pulsed with peptide was used as APCs for the $\mathrm{CD} 8^{+}$fraction in MLPC. After 8 days of MLPC culture, the T2-cell line pulsed with peptide was used as APCs in the ELISpot. IFN $\gamma$ and GrB ELISpot assays were performed as previously described $(22,27)$. Fig. 2 shows an exemplary ELISpot detecting specific CTL response of an analyzed lung cancer patient.

\section{Results}

Specific $T$ cell response to different TAAs. Due to the HLA restriction of the peptide used in our analyses, 14 (42\%) of 33 screened patients were eligible for further experiments after determining HLA-A*0201 positivity by FACS analysis (Fig. 1).

Due to the restriction of patient material, not all peptides could be analyzed for each patient (HER2, $n=10 ; G 250, n=12$; hTERT; survivin; G250; MAGE-A3 and AURA A1/B1, $\mathrm{n}=13$ each; Table II). Using interferon $\gamma$ (IFN $\gamma$ ) and granzyme B (GrB) ELISpot assays, specific cytotoxic T cell responses against at least one of these TAAs could be detected in 13/14 patients (93\%). In one patient diagnosed as adenocarcinoma, no specific immune responses against any TAAs could be detected (data not shown). Furthermore, $11 / 14(79 \%)$ patients exhibited $\mathrm{T}$ cell responses against at least 2 TAAs (data not shown). The most frequent specific CTL responses were detected against G250 (IFN $\gamma 58 / \mathrm{GrB}$, $58 \%)$ and PRAME (57/43\%) followed by hTERT (31/23\%) and RHAMM (29/29\%). Lower frequency was measured against the 2 MAGE-A3 peptides ( $23 / 8 \%$ and $31 / 31 \%$, respectively), survivin (23/23\%) and WT1 (15/7\%; Table III). Specific $\mathrm{T}$ cell responses were also detected against novel peptides
Table II. Peptides used for of specific T cell response against different TAAs.

\begin{tabular}{llc}
\hline Peptide & \multicolumn{1}{c}{ Sequence } & Position \\
\hline PRAME-P3 & ALYVDSLFFL & $300-309$ \\
RHAMM-R3 & ILSLELMKL & $165-173$ \\
G250 & QLLLSLLLL & $24-32$ \\
WT1 & RMFPNAPYL & $126-134$ \\
Aura A1 & TLCGTLDYL & $288-296$ \\
Aura B1 & KIADFGWSV & $215-223$ \\
Survivin & ELTLGEFLKL & $95-104$ \\
hTERT & ILAKFLHWL & $540-548$ \\
HER2-2 & RLLQETELV & $689-697$ \\
MAGE-A3_01 & FLWGPRALV & $271-279$ \\
MAGE-A3_02 & KVAELVHFL & $112-120$ \\
CMV & NLVPMVATV & $\mathbf{6 5 - 7 3}$ \\
IMP & GILGFVFTL & $\mathbf{5 8 - 6 6}$ \\
\hline
\end{tabular}

Amino acid sequence and the position in the polypeptide chain. Peptides shown in bold were used as positive controls. TAAs, tumorassociated antigens; PRAME, preferentially expressed antigen in melanoma; RHAMM, receptor for hyaluronic acid-mediated motility. Aura A1, Aurora kinase A; Aura B1, Aurora kinase B; MAGE-A3, melanoma-associated antigen 3.

from the Aurora kinases A and B. These peptides, Aura A1 and Aura B1, showed specific T cell responses in 36/29\% and $29 / 43 \%$ of patients, respectively. 
Table III. Specific T cell responses measured by IFN $\gamma$ and GrB ELISpot.

\begin{tabular}{llll}
\hline Peptide & \multicolumn{1}{c}{ Sequence } & IFN $\gamma(\%)$ & GrB (\%) \\
\hline PRAME-P3 & ALYVDSLFFL & $8 / 14(57)$ & $6 / 14(43)$ \\
RHAMM-R3 & ILSLELMKL & $4 / 14(29)$ & $4 / 14(29)$ \\
G250 & QLLLSLLLL & $7 / 12(58)$ & $7 / 12(58)$ \\
WT1 & RMFPNAPYL & $2 / 14(15)$ & $1 / 14(7)$ \\
Aura A1 & TLCGTLDYL & $4 / 13(31)$ & $3 / 13(23)$ \\
Aura B1 & KIADFGWSV & $4 / 13(31)$ & $5 / 13(38)$ \\
Survivin & ELTLGEFLKL & $3 / 13(23)$ & $3 / 13(23)$ \\
hTERT & ILAKFLHWL & $4 / 13(31)$ & $3 / 13(23)$ \\
HER2-2 & RLLQETELV & $5 / 10(50)$ & $0 / 10(0)$ \\
MAGE-A3_01 & FLWGPRALV & $3 / 13(23)$ & $1 / 13(8)$ \\
MAGE-A3_02 & KVAELVHFL & $4 / 13(31)$ & $4 / 13(31)$
\end{tabular}

Frequency of $\mathrm{T}$ cell responses calculated for each peptide. The most frequent specific CTL responses were detected against G250 and PRAME, followed by hTERT and RHAMM. Lower frequency was measured against the two MAGE-A3 peptides, survivin, and WT1. Specific $\mathrm{T}$ cell responses were also detected against novel peptides from the Aurora kinases A and B. IFN $\gamma$, interferon $\gamma$; ELISpot, enzyme-linked immunospot; PRAME, preferentially expressed antigen in melanoma; RHAMM, receptor for hyaluronic acid-mediated motility. Aura A1, Aurora kinase A; Aura B1, Aurora kinase B. MAGE-A3, melanoma-associated antigen 3.

Regarding identification of simultaneous CTL response, IFN $\gamma$ ELISpot showed that all patients with specific T cell response for epitopes of RHAMM and hTERT also had a CTL response against PRAME. Second, in all patient samples with CTL response against Aura A1, a reaction against the peptide Aura B1 was also detected (Fig. 3).

\section{Discussion}

In the treatment of lung cancer, significant developments have been made towards new therapeutic strategies. One promising option is the use of tumor vaccination. In our study, we demonstrated that peptides derived from different TAAs induce specific CTLs in a high percentage of patients with advanced lung cancer. It is necessary to identify novel peptides that can be used for targeted immunotherapy.

In most of the patients analyzed, specific CTL responses were detected. The methods that we used and the read out of parameters are well established. For both IFN $\gamma$ and GrB ELISpot results were mostly similar. Different results were detected only for HER2-2 with a trend to significance $(p=0.06)$. GrB ELISpot directly measures release of a cytolytic protein of these activated specific CTLs, while IFN $\gamma$ ELISpot detects the key cytokine in the complex process of $\mathrm{T}$ cell response. One reason for the different results in both ELISpots regarding the HER2 peptide may be that activation with this peptide does lead to a specific $\mathrm{T}$ cell response but not to a lytic activity of CTL.

The most frequent specific CTL response was detected for peptides derived from the known TAAs G250/CAIX and
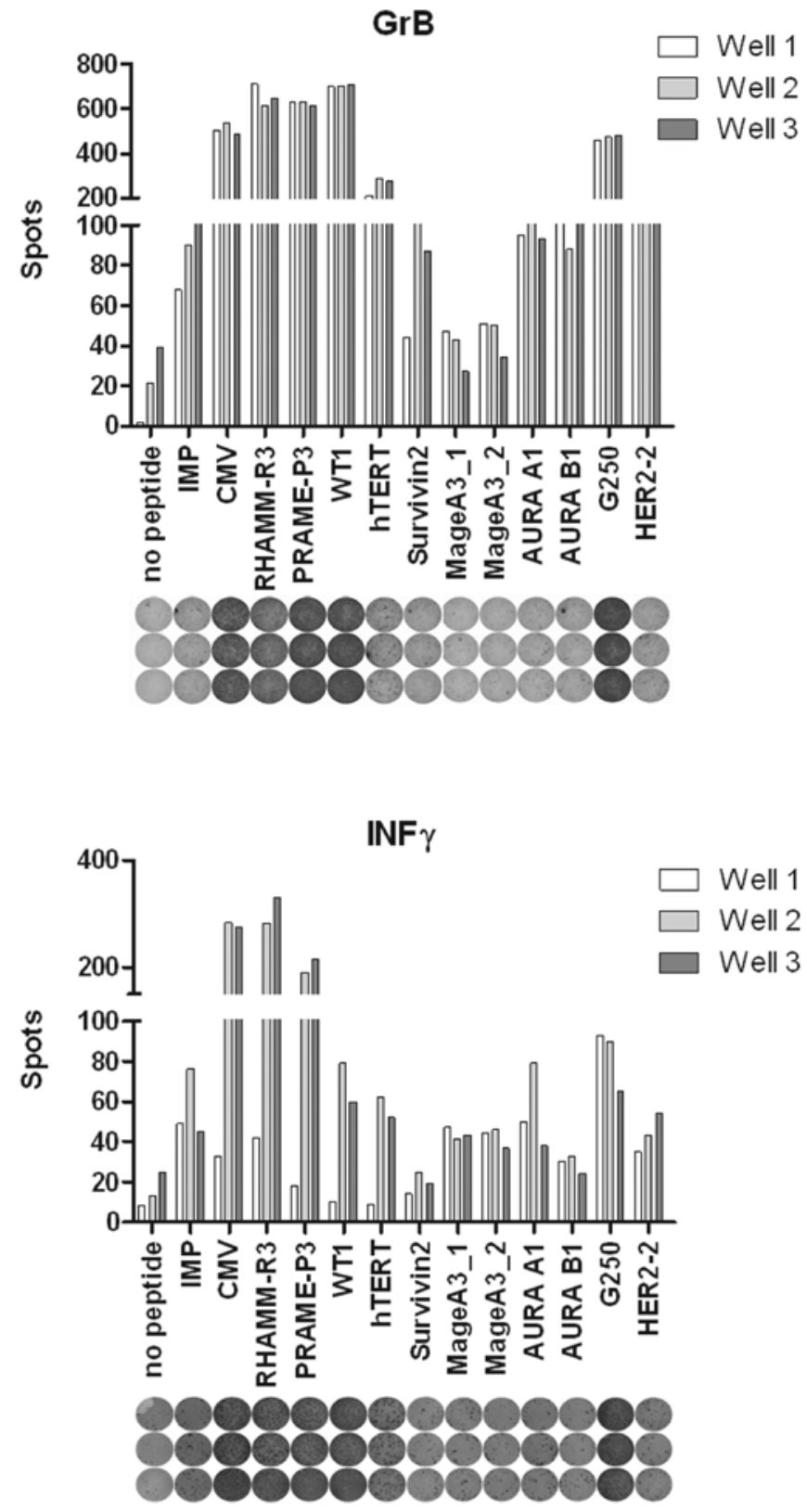

Figure 2. An exemplary ELISpot detecting specific T cell responses of CD8 ${ }^{+}$ $\mathrm{T}$ cells in a patient with metastatic lung cancer. Positive $\mathrm{T}$ cell responses were detected against the tumor-associated antigens PRAME, WT1, hTERT and G250 with a strong intensity. IMP and CMV were used as positive controls. ELISpot, enzyme-linked immunospot; PRAME, preferentially expressed antigen in melanoma.

PRAME. The carbonic anhydrase IX, G250/CAIX, is a well known TAA, particularly in solid tumors such as renal cell carcinoma. Specific $\mathrm{T}$ cell response of $\mathrm{CD}^{+} \mathrm{T}$ cells after activating PBMCs with the G250/CAIX derived peptide could be seen in patients with squamous cell cancer of head and neck. In 3/4 patients, specific CTL response was detected by GrB ELISpot, 1/7 by IFN $\gamma$ ELISpot respectively, (23). In lung cancer, there are different expression rates ranging from 75 to $95 \%(28,29)$. For renal cancer, clinical trials have been carried out using a monoclonal antibody against G250 $(30,31)$; however, peptide vaccinations are not yet in clinical testing. Our data indicate that this peptide derived from G250/CAIX could be a useful target for tumor vaccination in lung cancer. 


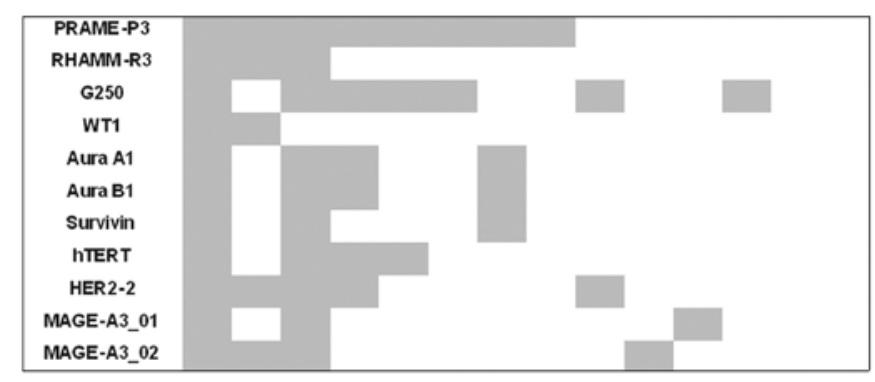

Figure 3. Simultaneous immune responses against peptides derived from different tumor antigens. Comparison of simultaneous CTL responses against a panel of immunogenic peptides tested in ELISpot analyses for IFN $\gamma$ production. Grey fields demonstrate a positive result in ELISpot analysis for interferon $\gamma$. Most patients showed simultaneous immune responses against several peptides and at least against one of these TAAs in 13/14 (93\%) patients; 11/14 (79\%) patients exhibited $\mathrm{T}$ cell responses against at least 2 TAAs. CTL, cytotoxic T cell (CTL); ELISpot, enzyme-linked immunospot; IFN $\gamma$, interferon $\gamma$; TAAs, tumor-associated antigens.

Second, the preferentially expressed antigen in melanoma (PRAME) derived peptide P3 induced a high CTL activity in the examined lung cancer patients which was confirmed in ELISpot assays. PRAME plays a crucial role in the inhibition of cell differentiation and apoptosis by repressing retinoic receptor signaling and showed a correlation with poor prognosis in high stage neuroblastomas $(32,33)$. In previous experiments by our group, the peptide PRAME-P3 induced a specific CTL response in $60 \%$ of AML patients $(n=10)$ (11). PRAME is expressed in several tumor entities, including lung cancer in up to $50 \%$ (34), making it a notable target for immunotherapeutic therapies. There is an ongoing phase I trial testing a new anticancer vaccine for patients with PRAME-positive NSCLC after tumor surgery (30).

Receptor for hyaluronic acid-mediated motility (RHAMM, CD168) has been defined as a leukemia-associated antigen and various RHAMM-derived HLA-A2-restricted peptides were tested for their immunogenicity by our group. In previous experiments, we generated RHAMM-R3-specific cytotoxic $\mathrm{T}$ cells of the peripheral blood from 7 of 13 AML patients. These cells were able to lyse RHAMM-R3 pulsed T2 cells and patient dendritic cells and were even able to lyse untreated AML blasts. It was also shown that RHAMM-R3 is a naturally processed epitope presented on HLA A2. Therefore, a phase I vaccination trial was initiated in which patients developed specific CTL response after 4 vaccinations (22). Similar results were obtained in patients with chronic lymphocytic leukemia and tumors of the head/neck region (35).

Since there are no clear data regarding overexpression of RHAMM in lung cancer, further analyses must be conducted.

Aside from these frequent responses against generally well known TAAs, we also analyzed specific $\mathrm{T}$ cell response against TAAs that are well known especially in lung cancer, such as hTERT and MAGE-A3. Human telomerase reverse transcriptase (hTERT) is the catalytic subunit of the human telomerase. Data have shown that it is overexpressed in lung cancer in up to $85 \%$ of lung cancer patients (36). Studies have also demonstrated that hTERT overexpression in lung cancer correlates with poor prognosis $(37,38)$. Trials evaluating the immunological and clinical response of a peptide vaccination with the optimized peptide telomerase reverse transcriptase p572Y (TERT572Y) showed that CTL response was detected in $76.2 \%$ of 21 patients after first vaccination and in $90.9 \%$ of 11 patients after the sixth vaccination. An early immunological response correlated with prolonged time to progression and overall survival (17). In our in vitro experiments, a specific CTL response was detected in $31 / 23 \%$. In contrast to our results, Bolonaki et al (17) could not measure any specific T cell response by IFN $\gamma$ ELISpot or pentamer staining before the first vaccination of lung cancer patients. Analysis of more patients will provide further information on the immunogenicity of hTERT peptides. Furthermore, a comparison between CTL response and hTERT expression in tumor tissue is warranted.

Melanoma-associated antigen 3 (MAGE-A3) is expressed in up to $55 \%$ of the NSCLC. The rate of expression is correlated with the stage of the disease and a poor prognosis (39). We examined specific $\mathrm{T}$ cell response for the peptides MAGE-A3_01 and MAGE-A3_02 and detected IFN $\gamma$ production against MAGE-A3_01 in 23\% and against MAGE-A3_02 in $31 \%$ of the analyzed patients. It could be demonstrated that specific T cells against MAGE-A3_01 generated from healthy volunteers recognize lung carcinoma cells (40). In previous investigations, 182 NSCLC patients with MAGE-A3 positive tumors demonstrated a positive trend for activity of MAGE-A3 protein vaccine with a relative improvement of DFI and DFS (41). Based on these data, an ongoing randomized phase III trial, MAGRIT, investigates the efficacy of MAGE-A3 tumor vaccination agents in preventing cancer relapse, when administered after tumor resection in patients with MAGE-A3-positive stages IB, II, and IIIA NSCLC (19).

Additionally, we analyzed $T$ cell responses against novel peptides. In our experiments, we used the Aurora kinase derived peptides Aura A1 and B1 which also induced specific CTL response at a lower level (30\%).

To date, 3 Aurora kinases are identified in mammals. Aurora kinases are serine/threonine kinases which play an essential role in cell mitosis and cell division. Aurora kinase A (Aura A) is localized in the centromers of interphase cells and is essential for formation of mitotic spindle. An ectopic expression may lead to malignant transformation of tissue. Its overexpression has been detected in several tumor tissues, including lung cancer. It could be demonstrated that its overexpression correlates with different histological subtypes of lung cancer, but does not indicate poorer prognosis (42). Furthermore, there are hints that an overexpression of Aura A is associated with drug resistance of tumor cells (43). Aurora kinase B (Aura B) is known as the chromosomal passenger protein and is localized in centromers of the prophase to the metaphase-anaphase transition. It is then localized to the midzone spindle during telophase and thereafter to midbody during cytokinesis. Aura B is overexpressed in lung cancer and an excessive overexpression correlates with poorer prognosis (44). Their frequent expression in lung cancer but also their ability to induce a specific immune response indicates that Aurora kinases could be novel targets in vaccination strategies.

Comparing immune responses against the different peptides could show a simultaneous CTL response in several cases. Regarding IFN $\gamma$ production, in all patient samples which showed an IFN $\gamma$ production against RHAMM and 
hTERT, response against PRAME could also be measured. One explanation for this could be a frequently occurring co-expression of different antigens in lung cancer, which has not yet been described. Recent published data verified a common co-expression of PRAME and MAGE-A3 in tumor tissue of lung cancer patients, which may have a poor prognostic value in some tumor stages (45). Further analysis of more lung cancer patient samples will provide insight into further epitopes leading to the discovery of usable peptides for polyvalent immunization with stronger efficacy comparing single peptide vaccinations.

Additionally, we observed a simultaneous occurrence of CTL response for the two Aurora kinase peptides, Aura A1 and Aura B1. Immunohistochemical analyses of a working group displayed a co-expression of both Aurora kinases in 33 patients with advanced lung cancer (46). A frequent co-expression in lung cancer could make them feasible targets for multipeptide vaccination as well. Furthermore, both Aurora kinases share a significant homology in their catalytic domain (47). An immunogenic peptide, which is found in both kinases, could enlarge the attack surface of a vaccination strategy.

In conclusion, specific $\mathrm{T}$ cell responses against several TAAs could be detected for the antigens hTERT, PRAME, G250 and RHAMM in high frequency in patients with lung cancer as well as in lower frequency against epitopes from other antigens. Moreover, novel immunogenic targets such as Aurora kinases A and B have been identified as immunogenic targets in lung cancer. Therefore, several antigen structures are appropriate candidates for immunotargeted approaches in lung cancer patients. Further analysis concerning the relationship between specific CTL response against different tumor antigen peptides and histology, clinical status of the patients and response to therapy, is mandatory. Furthermore, comparison of TAA expression in tumor tissue and specific cytotoxic $\mathrm{T}$ cell response will lead to a better understanding of the disease in an immunological manner and may extract patients that are appropriate candidates for tumor vaccination.

\section{Acknowledgements}

This study was supported by generous grants from the German José Carreras Leukemia Foundation (DJCLS A 10/01), the German Research Foundation (DFG GR2676/3-1) and the Else Kröner-Fresenius-Stiftung (2011-A63).

\section{References}

1. Sandler A, Gray R, Perry MC, et al: Paclitaxel-carboplatin alone or with bevacizumab for non-small-cell lung cancer. N Engl J Med 355: 2542-2550, 2006.

2. Cappuzzo F, Ciuleanu T, Stelmakh L, et al: Erlotinib as maintenance treatment in advanced non-small-cell lung cancer: a multicentre, randomised, placebo-controlled phase 3 study. Lancet Oncol 11: 521-529, 2010.

3. Kwak EL, Bang YJ, Camidge DR, et al: Anaplastic lymphoma kinase inhibition in non-small-cell lung cancer. N Engl J Med 363: 1693-1703, 2010.

4. Raez LE, Fein S and Podack ER: Lung cancer immunotherapy. Clin Med Res 3: 221-228, 2005.

5. Lynch TJ, Bondarenko I, Luft A, et al: Ipilimumab in combination with paclitaxel and carboplatin as first-line treatment in stage IIIB/IV non-small-cell lung cancer: results from a randomized, double-blind, multicenter phase II study. J Clin Oncol 30 2046-2054, 2012.
6. Topalian SL, Hodi FS, Brahmer JR, et al: Safety, activity, and immune correlates of anti-PD-1 antibody in cancer. N Engl J Med 366: 2443-2454, 2012.

7. Marigo I, Dolcetti L, Serafini P, Zanovello P and Bronte V: Tumor-induced tolerance and immune suppression by myeloid derived suppressor cells. Immunol Rev 222: 162-179, 2008.

8. Pardoll D: Does the immune system see tumors as foreign or self? Annu Rev Immunol 21: 807-839, 2003.

9. Novellino L, Castelli C and Parmiani G: A listing of human tumor antigens recognized by T cells: March 2004 update. Cancer Immunol Immunother 54: 187-207, 2005.

10. Greiner J, Bullinger L, Guinn BA, Döhner H and Schmitt M: Leukemia-associated antigens are critical for the proliferation of acute myeloid leukemia cells. Clin Cancer Res 14: 7161-7166, 2008.

11. Greiner J, Schmitt M, Li L, et al: Expression of tumor-associated antigens in acute myeloid leukemia: implications for specific immunotherapeutic approaches. Blood 108: 4109-4117, 2006.

12. Ribas A, Butterfield LH, Glaspy JA and Economou JS: Current developments in cancer vaccines and cellular immunotherapy. J Clin Oncol 21: 2415-2432, 2003.

13. Rosenberg SA: Development of effective immunotherapy for the treatment of patients with cancer. J Am Coll Surg 198: 685-696, 2004.

14. Banchereau J and Steinman RM: Dendritic cells and the control of immunity. Nature 392: 245-252, 1998.

15. Kelly RJ, Gulley JL and Giaccone G: Targeting the immune system in non-small-cell lung cancer: bridging the gap between promising concept and therapeutic reality. Clin Lung Cancer 11: 228-237, 2010.

16. Gridelli C, Rossi A, Maione P, Ferrara ML, Castaldo V and Sacco PC: Vaccines for the treatment of non-small cell lung cancer: a renewed anticancer strategy. Oncologist 14: 909-920, 2009.

17. Bolonaki I, Kotsakis A, Papadimitraki E, et al: Vaccination of patients with advanced non-small-cell lung cancer with an optimized cryptic human telomerase reverse transcriptase peptide. J Clin Oncol 25: 2727-2734, 2007.

18. Peled N, Oton AB, Hirsch FR and Bunn P: MAGE A3 antigenspecific cancer immunotherapeutic. Immunotherapy 1: 19-25, 2009.

19. Tyagi P and Mirakhur B: MAGRIT: the largest-ever phase III lung cancer trial aims to establish a novel tumor-specific approach to therapy. Clin Lung Cancer 10: 371-374, 2009.

20. Eaton KD and Martins RG: Maintenance chemotherapy in non-small cell lung cancer. J Natl Compr Canc Netw 8: 815-821, 2010.

21. Walter S, Weinschenk T, Stenzl A, et al: Multipeptide immune response to cancer vaccine IMA901 after single-dose cyclophosphamide associates with longer patient survival. Nat Med 18: 1254-1261, 2012.

22. Schmitt M, Schmitt A, Rojewski MT, et al: RHAMM-R3 peptide vaccination in patients with acute myeloid leukemia, myelodysplastic syndrome, and multiple myeloma elicits immunologic and clinical responses. Blood 111: 1357-1365, 2008.

23. Greiner J, Li L, Ringhoffer M, et al: Identification and characterization of epitopes of the receptor for hyaluronic acid-mediated motility (RHAMM/CD168) recognized by $\mathrm{CD} 8^{+} \mathrm{T}$ cells of HLA-A2-positive patients with acute myeloid leukemia. Blood 106: 938-945, 2005.

24. Greiner J, Dohner H and Schmitt M: Cancer vaccines for patients with acute myeloid leukemia - definition of leukemia-associated antigens and current clinical protocols targeting these antigens. Haematologica 91: 1653-1661, 2006.

25. Bellantuono I, Gao L, Parry S, et al: Two distinct HLA-A0201presented epitopes of the Wilms tumor antigen 1 can function as targets for leukemia-reactive CTL. Blood 100: 3835-3837, 2002.

26. Parkhurst MR, Riley JP, Igarashi T, Li Y, Robbins PF and Rosenberg SA:Immunization of patients with the hTERT:540-548 peptide induces peptide-reactive $\mathrm{T}$ lymphocytes that do not recognize tumors endogenously expressing telomerase. Clin Cancer Res 10: 4688-4698, 2004.

27. Greiner J, Schmitt A, Giannopoulos K, et al: High-dose RHAMM-R3 peptide vaccination for patients with acute myeloid leukemia, myelodysplastic syndrome and multiple myeloma. Haematologica 95: 1191-1197, 2010.

28. Swinson DE, Jones JL, Richardson D, et al: Carbonic anhydrase IX expression, a novel surrogate marker of tumor hypoxia, is associated with a poor prognosis in non-small-cell lung cancer. J Clin Oncol 21: 473-482, 2003. 
29. Vermylen P, Roufosse C, Burny A, et al: Carbonic anhydrase IX antigen differentiates between preneoplastic malignant lesions in non-small cell lung carcinoma. Eur Respir J 14: 806-811, 1999.

30. ClinicalTrials.gov: http://www.clinicaltrials.gov/. H-Cg. NCT00003102.

31. Siebels M, Rohrmann K, Oberneder R, et al: A clinical phase I/II trial with the monoclonal antibody cG250 (RENCAREX $\left.{ }^{\circledR}\right)$ and interferon-alpha-2a in metastatic renal cell carcinoma patients. World J Urol 29: 121-126, 2011.

32. Epping MT, Wang L, Edel MJ, Carlée L, Hernandez M and Bernards R: The human tumor antigen PRAME is a dominant repressor of retinoic acid receptor signaling. Cell 122: 835-847, 2005.

33. Oberthuer A, Hero B, Spitz R, Berthold F and Fischer M: The tumor-associated antigen PRAME is universally expressed in high-stage neuroblastoma and associated with poor outcome. Clin Cancer Res 10: 4307-4313, 2004.

34. Kessler JH, Beekman NJ, Bres-Vloemans SA, et al: Efficient identification of novel HLA-A*0201-presented cytotoxic T lymphocyte epitopes in the widely expressed tumor antigen PRAME by proteasome-mediated digestion analysis. J Exp Med 193: 73-88, 2001

35. Schmitt A, Barth TF, Beyer E, et al: The tumor antigens RHAMM and G250/CAIX are expressed in head and neck squamous cell carcinomas and elicit specific $\mathrm{CD}^{+} \mathrm{T}$ cell responses. Int $\mathrm{J}$ Oncol 34: 629-639, 2009.

36. Albanell J, Lonardo F, Rusch V, et al: High telomerase activity in primary lung cancers: association with increased cell proliferation rates and advanced pathologic stage. J Natl Cancer Inst 89: 1609-1615, 1997

37. Wang L, Soria JC, Kemp BL, Liu DD, Mao L and Khuri FR: hTERT expression is a prognostic factor of survival in patients with stage I non-small cell lung cancer. Clin Cancer Res 8 : 2883-2889, 2002.

38. Fujita Y, Fujikane T, Fujiuchi S, et al: The diagnostic and prognostic relevance of human telomerase reverse transcriptase mRNA expression detected in situ in patients with nonsmall cell lung carcinoma. Cancer 98: 1008-1013, 2003.
39. Gure AO, Chua R, Williamson B, et al: Cancer-testis genes are coordinately expressed and are markers of poor outcome in non-small cell lung cancer. Clin Cancer Res 11: 8055-8062, 2005.

40. Eifuku R, Takenoyama M, Yoshino I, et al: Analysis of MAGE-3 derived synthetic peptide as a human lung cancer antigen recognized by cytotoxic T lymphocytes. Int J Clin Oncol 6: 34-39, 2001.

41. Vansteenkiste J, Zielinski M, Linder A, Dahabre J, Esteban E, Malinowski W, Jassem J, Passlick B, Lehmann F and Brichard VG: Final results of a multi-center, double-blind, randomized, placebo-controlled phase II study to assess the efficacy of MAGE-A3 immunotherapeutic as adjuvant therapy in stage IB/II non-small cell lung cancer (NSCLC). J Clin Oncol 25: $7554,2007$.

42. Lo Iacono M, Monica V, Saviozzi S, et al: Aurora Kinase A expression is associated with lung cancer histological-subtypes and with tumor de-differentiation. J Transl Med 9: 100, 2011.

43. Anand S, Penrhyn-Lowe S and Venkitaraman AR: AURORA-A amplification overrides the mitotic spindle assembly checkpoint, inducing resistance to Taxol. Cancer Cell 3: 51-62, 2003.

44. Smith SL, Bowers NL, Betticher DC, et al: Overexpression of aurora B kinase (AURKB) in primary non-small cell lung carcinoma is frequent, generally driven from one allele, and correlates with the level of genetic instability. Br J Cancer 93: 719-729, 2005.

45. Linder A, Budihardjo-Welim H, Velehorschi W, Coche T, Gruselle O, D'Agostino D, Louahed J, Lehmann F, Brichard VG; Lungenklinik Hemer, Hemer Nordrhein-Westfalen, Germany; Praxis für Pathologie Hemer, Hemer, Germany; GlaxoSmithKline Biologicals, Rixensart, Belgium: Prognostic value of MAGE-A3 and PRAME gene expression in non-small-cell lung cancer (NSCLC). J Clin Oncol 30: 7056, 2012.

46. Gautschi O, Heighway J, Mack PC, Purnell PR, Lara PN Jr and Gandara DR: Aurora kinases as anticancer drug targets. Clin Cancer Res 14: 1639-1648, 2008.

47. Fu J, Bian M, Jiang Q and Zhang C: Roles of Aurora kinases in mitosis and tumorigenesis. Mol Cancer Res 5: 1-10, 2007. 